\title{
microRNA-20b contributes to high glucose-induced podocyte apoptosis by targeting SIRT7
}

\author{
XIAOJING WANG ${ }^{1}$, BO LIN ${ }^{2}$, LIN NIE $^{3}$ and PING LI ${ }^{1}$ \\ ${ }^{1}$ Department of Endocrinology, Yuncheng Central Hospital, Yuncheng, Shanxi 044000; \\ ${ }^{2}$ Henan University School of Basic Medical Sciences, Kaifeng, Henan 475004; \\ ${ }^{3}$ Department of Endocrinology, Beijing Airport Hospital, Beijing 101318, P.R. China
}

Received September 24, 2016; Accepted June 15, 2017

DOI: $10.3892 / \mathrm{mmr} .2017 .7224$

\begin{abstract}
Previous reports have indicated that microRNAs (miRNAs) have an important role in the pathogenesis of diabetic nephropathy (DN). Podocyte apoptosis induced by high glucose (HG) is characteristic of DN. However, the role of miRNAs in HG-induced podocyte apoptosis remains poorly understood. The present study investigated the role and potential underlying mechanism of miRNA-20b (miR-20b) in podocyte apoptosis induced by HG. The results demonstrated that miR-20b was significantly upregulated in HG-treated podocytes, as determined by reverse transcription-quantitative polymerase chain reaction (RT-qPCR). Caspase-3 activity and TUNEL assays indicated that suppression of miR-20b using miR-20b inhibitors significantly inhibited the podocyte apoptosis induced by HG. Sirtuin 7 (SIRT7) was identified as a functional target of miR-20b by a Dual-Luciferase activity reporter assay, RT-qPCR and western blot analysis. Silencing SIRT7 promoted HG-induced podocyte apoptosis, as determined by the caspase-3 activity, while SIRT7 overexpression attenuated HG-induced podocyte apoptosis. However, SIRT7 silencing significantly blocked the protective effect of miR-20b suppression against $\mathrm{HG}$-induced apoptosis. In conclusion, these results indicate that miR-20b may contribute to $\mathrm{HG}$-induced podocyte apoptosis by targeting SIRT7, providing a potential therapeutic target for the treatment of DN.
\end{abstract}

\section{Introduction}

Diabetic nephropathy (DN), a serious complication of diabetes, has emerged as the primary cause of end-stage

Correspondence to: Miss Xiaojing Wang, Department of Endocrinology, Yuncheng Central Hospital, 3690 Hedong Street, Yuncheng, Shanxi 044000, P.R. China

E-mail: wangxiaojingsx@163.com

Abbreviations: miRNA, microRNA; HG, high glucose; SIRT7, sirtuin 7; DN, diabetic nephropathy; UTR, untranslated region

Key words: diabetic nephropathy, podocyte, microRNA-20b, sirtuin 7 renal disease (1). DN severely affects the quality of life of patients (2). Therefore, there is an urgent demand for the development of novel therapies to prevent the progression of DN. Podocytes are a group of terminally differentiated cells that are attached to the outer surface of the glomerular basement membrane and function in maintaining the integrity of the glomerular basement membrane (3). Previous reports have indicated that podocytes have a pivotal role in the development and progression of DN $(4,5)$. High glucose (HG) induces podocyte apoptosis, which contributes to the pathogenesis of DN $(6,7)$. Therefore, targeting podocyte apoptosis may provide a novel therapeutic strategy for the treatment of DN.

Previous reports have indicated that microRNAs (miRNAs) function as regulators of gene expression. miRNAs inhibit protein translation by interacting with the 3'-untranslated region (UTR) of the mRNA of target genes $(8,9)$. Therefore, miRNAs are involved in the regulation of various cellular processes, including cell proliferation, survival, apoptosis and differentiation (10). Furthermore, an increasing number of studies have indicated that miRNAs participate in the pathogenesis of various diseases and represents novel biomarkers for diagnosis, prognosis and treatment (11-13). Certain miRNAs have been reported to be associated with the pathogenesis of DN, which highlights their potential as promising therapeutic targets for DN $(14,15)$. In addition, recent studies have demonstrated that several miRNAs, including miRNA-34c (miR-34c), miR-30a and miR-218, are aberrantly expressed in podocytes induced by $\mathrm{HG}$ and have important roles in regulating podocyte apoptosis $(16,17)$. However, the exact role of miRNAs in podocyte apoptosis remains largely unknown.

The sirtuin family of proteins consists of seven members (SIRT1-7), which are nicotinamide adenine dinucleotide oxidized form-dependent deacetylases that have important roles in the regulation of various biological processes, including metabolism, DNA repair, inflammation, stress response, cell cycle and apoptosis $(18,19)$. Sirtuin 7 (SIRT7) is the most recently characterized sirtuin, however, important roles for SIRT7 in several pathological processes have been recently reported (20). SIRT7 regulates lipid metabolism, chromatin remodeling and protein synthesis (20). SIRT7 has been reported to have a key role in cellular survival in response to a variety of stress conditions, including hypoxia, and endoplasmic reticulum and genomic stress (21-23). However, 
whether SIRT7 regulates podocyte apoptosis remains to be established.

It has been hypothesized that miR-20b may be an miRNA that is associated with apoptosis (24). Recently, miR-20b was reported to be a response gene under hyperglycemic conditions in retinal endothelial cells (25). However, whether miR-20b participates in podocyte apoptosis under hyperglycemic conditions is yet to be determined. The present study aimed to investigate the biological role and potential underlying mechanism of miR-20b in podocyte apoptosis induced by HG. The results demonstrated that miR-20b was significantly upregulated in HG-treated podocytes. Suppression of miR-20b significantly inhibited podocyte apoptosis induced by HG. In addition, SIR7 was identified as a functional target of miR-20b that may contribute to the protective effect of miR-20b suppression on HG-induced apoptosis. These results indicate that miR-20b may contribute to HG-induced podocyte apoptosis by targeting SIRT7, therefore representing a potential therapeutic target for DN.

\section{Materials and methods}

Cell cultures and treatments. Conditionally immortalized mouse podocytes were purchased from the Cell Resource Center of Peking Union Medical College (Beijing, China). Podocytes were routinely cultured as previously described (26). Briefly, podocytes were cultured in RPMI-1640 medium (Gibco; Thermo Fisher Scientific, Inc., Waltham, MA, USA) containing 10\% fetal bovine serum (FBS; Gibco; Thermo Fisher Scientific, Inc.), $10 \mathrm{U} / \mathrm{ml}$ interferon- $\gamma$ (Sangon Biotech Co., Ltd., Shanghai, China), 100 U/ml Penicillin and $0.1 \mathrm{mg} / \mathrm{ml}$ Streptomycin (Sigma-Aldrich; Merck KGaA, Darmstadt, Germany) at $33^{\circ} \mathrm{C}$ in a $5 \% \mathrm{CO}_{2}$ atmosphere with relative humidity of $95 \%$. Once cells reached a confluence of $\sim 80 \%$, the cells were cultured in the medium described above without interferon- $\gamma$ for 14 days at $37^{\circ} \mathrm{C}$ in a $5 \% \mathrm{CO}_{2}$ atmosphere. Prior to experiments, the podocytes were cultured in serum-free medium for $24 \mathrm{~h}$ at $37^{\circ} \mathrm{C}$ in a $5 \% \mathrm{CO}_{2}$ atmosphere and relative humidity of $95 \%$ to synchronize cell growth. Subsequently, podocytes were cultured in serum-free medium containing $5,10,15,20,25$ or $30 \mathrm{mM}$ D-glucose for 6,12 or $24 \mathrm{~h}$ at $37^{\circ} \mathrm{C}$. HEK293T cells were purchased from the American Type Culture Collection (Manassas, VA, USA) and maintained in Dulbecco's modified Eagle's medium (Gibco; Thermo Fisher Scientific, Inc.) containing 10\% FBS and $100 \mathrm{U} / \mathrm{ml}$ Penicillin and $0.1 \mathrm{mg} / \mathrm{ml}$ Streptomycin in a humidified atmosphere with $5 \% \mathrm{CO}_{2}$ at $37^{\circ} \mathrm{C}$.

Cell transfection. miR-20b inhibitor (5'-cuaccugcacuaugagcacaaag-3'), miR-20b mimic (5'-caaagugcucauagugcagguag-3') and negative control (NC; 5'-agacugauuccgcgauaccaggc-3') were synthesized by Shanghai GenePharma Co., Ltd. (Shanghai, China) and transfected into cells at a final concentration of $30 \mathrm{nM}$ using Lipofectamine 2000 (Invitrogen; Thermo Fisher Scientific, Inc.) for $24 \mathrm{~h}$ at $37^{\circ} \mathrm{C}$. SIRT7 small interfering RNA (siRNA) and NC siRNA were purchased from Santa Cruz Biotechnology, Inc. (Dallas, TX, USA) and transfected into cells at a final concentration of $50 \mathrm{nM}$ using Lipofectamine 2000 (Invitrogen). For SIRT7 overexpression, the open reading frame of SIRT7 cDNA was inserted into
pcDNA3.1 plasmids (BioVector NTCC, Inc., Beijing, China) to generate pcDNA3.1/SIRT7 overexpressing vectors. The pcDNA3.1/SIRT7 vectors were transfected into cells at a final concentration of $1 \mu \mathrm{g} / \mathrm{ml}$ using Lipofectamine 2000. Empty pcDNA3.1 vectors were used for the control group. Following transfection for $24 \mathrm{~h}$, cells were subjected to the subsequent experiments.

RNA extraction and reverse transcription-quantitative polymerase chain reaction $(R T-q P C R)$. Total RNA was extracted by using TRIzol reagent (Invitrogen; Thermo Fisher Scientific, Inc.) according to the manufacturer's protocol. RT of mRNA and miRNA were performed by using M-MLV reverse transcriptase (Takara Biotechnology Co., Ltd., Dalian, China) or a One Step PrimeScript ${ }^{\circledR}$ miRNA cDNA Synthesis kit (Takara Biotechnology Co., Ltd.), respectively. For the RT of mRNA, a mixture of RNA, Oligo (dt) $)_{12-18}$ primer, dNTP mixture, M-MLV buffer (all from Takara Biotechnology Co., Ltd.), and M-MLV reverse transcriptase was incubated at $42^{\circ} \mathrm{C}$ for $1 \mathrm{~h}$ and then at $70^{\circ} \mathrm{C}$ for $15 \mathrm{~min}$. For the RT of miRNA, a mixture of RNA, miRNA Reaction Buffer Mix, Universal Adaptor Primer, and miRNA PrimeScript ${ }^{\circledR}$ RT Enzyme Mix (all from Takara Biotechnology Co., Ltd.) was incubated at $37^{\circ} \mathrm{C}$ for $60 \mathrm{~min}$ and $85^{\circ} \mathrm{C}$ for $5 \mathrm{sec}$. Gene expression was detected by using the Power SYBR Green PCR Master Mix (Applied Biosystems; Thermo Fisher Scientific, Inc.) on an Applied Biosystems AB7500 Real-Time PCR System (Applied Biosystems; Thermo Fisher Scientific, Inc.) under the following conditions: $95^{\circ} \mathrm{C}$ for $5 \mathrm{~min}$; 30 cycles of $94^{\circ} \mathrm{C}$ for $20 \mathrm{sec}, 55^{\circ} \mathrm{C}$ for $30 \mathrm{sec}$ and $72^{\circ} \mathrm{C}$ for $35 \mathrm{sec}$; and $72^{\circ} \mathrm{C}$ for $5 \mathrm{~min}$. The primers used were as follows: SIRT7, 5'-agaactgtgatgggetccac-3' (forward) and 5'-tgaagggcagtacgctcagt-3' (reverse); $\beta$-actin, 5'-ttccttcttgggtatggaat-3' (forward) and 5'-gagcaatgatcttgatcttc-3' (reverse); miR-20b, 5'-acactccagctgggcaaagtgctcatagtgc-3' (forward) and 5'-tggtgtcgtggagtcg-3' (reverse); and U6, 5'-tgcgggtgctcgcttcggcagc-3' (forward) and 5 '-ccagtgcagggtccgaggt-3' (reverse). $\beta$-actin or U6 were used as the internal controls for normalization. Data were obtained from three independent experiments. Gene expression was calculated by using $2^{-\Delta \Delta \mathrm{Cq}}(27)$, normalized against $\beta$-actin or U6 and compared with the control group.

Caspase-3 activity assay. Caspase-3 activity was measured using a Caspase-3 Activity assay kit (Beyotime Institute of Biotechnology, Haimen, China), according to the manufacturer's protocol. Briefly, $2 \times 10^{6}$ cells were lysed using radioimmunoprecipitation assay buffer (Beyotime Institute of Biotechnology) and the supernatant was collected. The protein concentration was measured using Enhanced BCA Protein Assay kit (Beyotime Institute of Biotechnology). A total of $100 \mu \mathrm{g}$ protein was incubated with $5 \mu \mathrm{l}$ DEVD-pNA substrate $(4 \mathrm{mM})$ in $50 \mu \mathrm{l}$ reaction buffer at $37^{\circ} \mathrm{C}$ for $2 \mathrm{~h}$. The absorbance value at a wavelength of $405 \mathrm{~nm}$ was detected using an ELISA reader (BioTek Instruments, Inc., Winooski, VT, USA).

TUNEL assay. Cell apoptosis was detected by using Roche In Situ Cell Death Detection kit (Roche Diagnostics, Indianapolis, IN, USA), according to the manufacturer's protocol. Briefly, slides of $1 \times 10^{6}$ cells were fixed with $4 \%$ paraformaldehyde for 
A

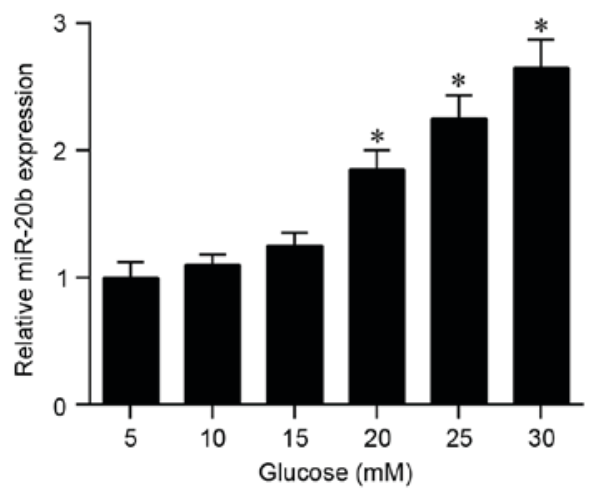

B

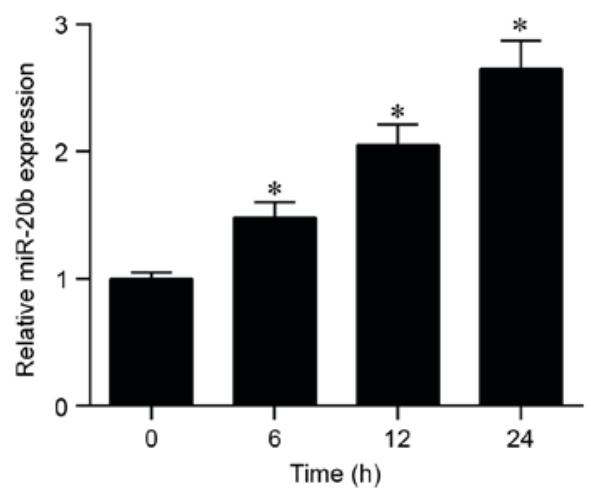

Figure 1. miR-20b is induced by high glucose in podocytes. (A) RT-qPCR detection of miR-20b expression in podocytes exposed to 5, 10, $15,20,25$ and 30 mM glucose for $24 \mathrm{~h}$. ${ }^{\mathrm{P}}<0.05$ vs. $5 \mathrm{mM}$. (B) RT-qPCR detection of miR-20b expression in podocytes exposed to $30 \mathrm{mM}$ glucose for 6,12 and $24 \mathrm{~h}$. ${ }^{*} \mathrm{P}<0.05 \mathrm{vs} .0 \mathrm{~h}$. miR, microRNA; RT-qPCR, reverse transcription-quantitative polymerase chain reaction.

$30 \mathrm{~min}$ at room temperature and permeabilized with $0.2 \%$ Triton-X-100 for $5 \mathrm{~min}$ at room temperature. The cells were then incubated with $450 \mu 1$ TUNEL label solution and $50 \mu 1$ TUNEL enzyme solution at $37^{\circ} \mathrm{C}$ for $1 \mathrm{~h}$ in the dark. Subsequently, the slides were observed under a fluorescence microscope (Olympus Corporation, Tokyo, Japan). The number of apoptotic cells in five random fields per slide were counted and averaged.

Western blot analysis. Cells were harvested and lysed in radioimmunoprecipitation assay lysis buffer (Beyotime Institute of Biotechnology) and protein concentration was determined by a BCA kit (Beyotime Institute of Biotechnology). A total of $40 \mu \mathrm{g}$ protein was separated by $12 \%$ SDS-PAGE and electrotransferred to polyvinylidene difluoride membranes (EMD Millipore, Billerica, MA, USA). Following incubation with $3 \%$ nonfat milk for $1 \mathrm{~h}$ at $37^{\circ} \mathrm{C}$, the membranes were incubated with the following rabbit primary antibodies: Anti-SIRT7 (cat. no. ab78977; 1:250; Abcam, Cambridge, MA, USA) and anti- $\beta$-actin (cat. no. ab8227; $1: 1,000$; Abcam) at $4^{\circ} \mathrm{C}$ overnight. Subsequently, the membrane was washed thrice with Tris-buffered saline with $0.05 \%$ Tween- 20 and incubated with horseradish peroxidase-conjugated secondary antibodies (cat. no. bs-0295G-HRP; 1:1,000; BIOSS, Beijing, China) for $1 \mathrm{~h}$ at $37^{\circ} \mathrm{C}$. The immunoblots were visualized by a Pierce ECL Western Blotting Substrate (Pierce; Thermo Fisher Scientific, Inc.). Densitometric analysis of the protein bands was performed using Image-Pro Plus software version 6.0 (Media Cybernetics, Inc., Rockville, MD, USA).

Dual-Luciferase reporter assay. Bioinformatics analysis for miR-20b was performed using a web server of TargetScan: Prediction of microRNA targets (http://www.targetscan .org/). The 3'-untranslated region (UTR) of SIRT7 containing miR-20b binding sites was synthesized and cloned into the pmirGLO Dual-Luciferase miRNA Target Expression Vector (Promega Corporation, Madison, WI, USA). The 3'-UTR of SIRT7 containing mutations in the miR-20b recognition sites was synthesized by QuikChange Multi Site-Directed Mutagenesis kit (Agilent Technologies, Inc., Santa Clara, CA, USA) and cloned into the pmirGLO Dual-Luciferase miRNA Target Expression Vector. The constructed pmirGLO vectors were cotransfected into HEK293T cells with miR-20b mimics
(30 nM) or NC mimics (30 nM) by using Lipofectamine 2000. Following transfection for $48 \mathrm{~h}$, the cells were immediately harvested and subjected to detection of luciferase activity. the relative luciferase activity was analyzed by using a Dual-Glo Luciferase assay system (Promega Corporation), according to the manufacturer's protocol. Relative luciferase activity was calculated according to the formula: Firefly luciferase/Renilla luciferase.

Statistical analysis. Quantitative data are presented as the mean + standard deviation. Statistical analyses were performed by one-way analysis of variance followed by the Bonferroni post-hoc test using SPSS software version 11.5 (SPSS, Inc., Chicago, IL, USA). $\mathrm{P}<0.05$ was considered to indicate a statistically significant difference.

\section{Results}

miR-20b expression is upregulated by HG in podocytes. To investigate the relevance of $\mathrm{miR}-20 \mathrm{~b}$ in podocytes, the present study determined the expression pattern of miR-20b in podocytes exposed to $\mathrm{HG}$ in vitro by RT-qPCR. As demonstrated in Fig. 1A, the expression level of miR-20b was significantly and dose-dependently upregulated in podocytes exposed to concentrations of glucose $>20 \mathrm{mM}$ after $24 \mathrm{~h}$ treatment, compared with treatment with $5 \mathrm{mM}$ glucose. Furthermore, miR-20b was significantly increased after 6, 12 and $24 \mathrm{~h}$ treatment with $30 \mathrm{mM}$ glucose (Fig. 1B), compared with the $0 \mathrm{~h}$ treatment group. These results indicate that miR-20b may be involved in the response to HG in podocytes.

Suppression of miR-20b attenuates apoptosis of podocytes. To investigate the precise biological role of miR-20b in HG-induced podocyte apoptosis, the current study suppressed the expression of miR-20b by transiently transfecting podocytes with miR-20b inhibitor. The results demonstrated that miR-20b expression was significantly suppressed by miR-20b inhibitor transfection in cultured podocytes treated with $\mathrm{HG}$, compared with podocytes treated with HG only (Fig. 2A). The effect of miR-20b suppression on apoptosis was subsequently detected by TUNEL (Fig. 2B) and caspase-3 activity (Fig. 2C) assays. The results demonstrated 


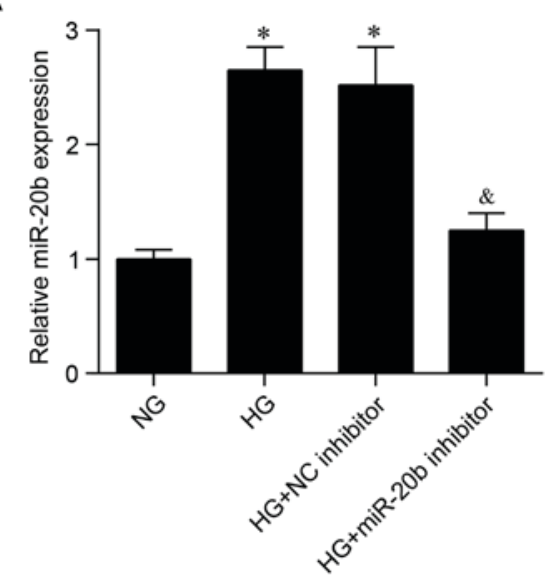

B

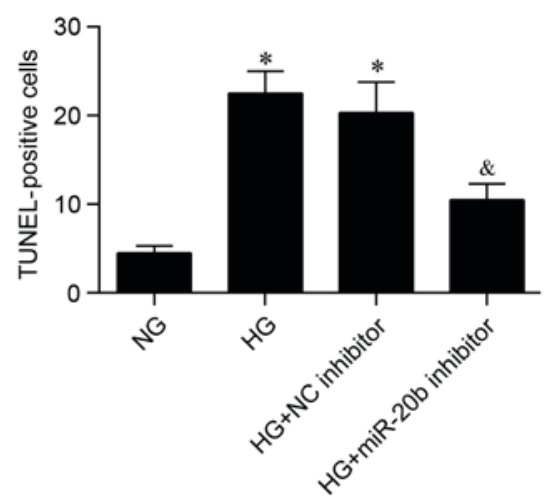

C

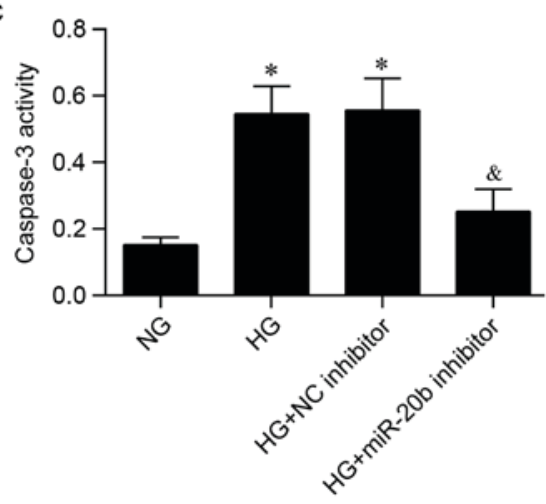

Figure 2. Suppression of miR-20b inhibits HG-induced podocyte apoptosis. Podocytes were treated with NG $(5 \mathrm{mM})$ or $\mathrm{HG}(30 \mathrm{mM})$ with or without transfection with miR-20b inhibitor or NC inhibitor for $24 \mathrm{~h}$. (A) miR-20b expression in different groups was examined by reverse transcription-quantitative polymerase chain reaction. Apoptosis of podocytes in different groups was detected by (B) TUNEL and (C) caspase-3 activity assays. " $\mathrm{P}<0.05$ vs. NG; ${ }^{\circledR} \mathrm{P}<0.05$ vs. $\mathrm{HG}$ and $\mathrm{HG}+\mathrm{NC}$ inhibitor. miR, microRNA; HG, high glucose; NG, normal glucose; $\mathrm{NC}$, negative control.

that HG exposure significantly induced podocyte apoptosis compared with cells treated with normal glucose $(5 \mathrm{mM})$, whereas suppression of miR-20b significantly prevented podocyte apoptosis induced by HG (Fig. 2B and C). These results indicate that miR-20b may be involved in podocyte apoptosis induced by HG.

SIRT7 is a target of miR-20b. To elucidate the underlying molecular mechanism by which miR-20b regulates podocyte apoptosis, the present study aimed to identify the potential target gene for miR-20b using bioinformatics

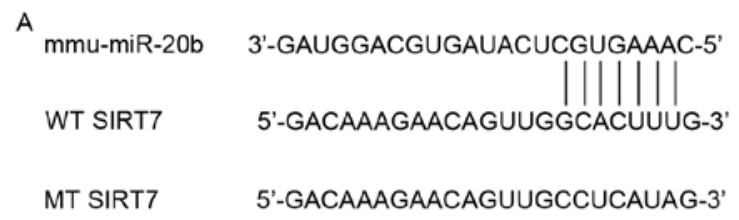

B

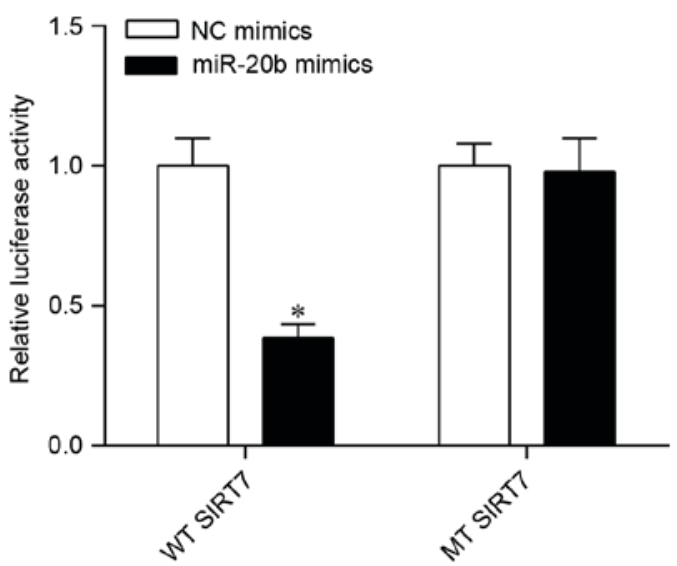

Figure 3. miR-20b targets the 3'-UTR of SIRT7. (A) miR-20b sequence and its target sequence in the 3'-UTR of SIRT7. (B) Relative luciferase activities of vector constructs carrying WT or MT SIRT7 3'-UTR in HEK293T cells cotransfected with miR-20b or NC mimics. The luciferase activity was detected by a Dual-Luciferase assay system following incubation for 48 h. " $\mathrm{P}<0.05$ vs. NC mimics. miR, microRNA; UTR, untranslated region; SIRT7, sirtuin 7; WT, wild-type; MT, mutant; NC, negative control.

analysis. Notably, SIRT7, an important stress adaptor molecule for cell survival, was identified as a putative target of miR-20b (Fig. 3A). To verify whether SIRT7 is a direct target gene of miR-20b, a Dual-Luciferase reporter assay was performed. Cotransfection of wild-type SIRT7 3'-UTR construct with miR-20b mimics into HEK293T cells resulted into a significant decrease in luciferase activity compared with cells cotransfected with NC mimics (Fig. 3B). However, miR-20b mimics exhibited no significant effect on the luciferase activity when cotransfected with mutant SIRT7 3'-UTR construct (Fig. 3B). The results indicate that miR-20b directly targets the 3'-UTR of SIRT7. To further confirm this prediction, the direct effect of miR-20b on SIRT7 expression was investigated. The results demonstrated that the mRNA and protein expression of SIRT7 was significantly upregulated by miR-20b suppression in podocytes treated with HG (Fig. 4). Taken together, these results indicate that SIRT7 is a direct target gene of miR-20b.

SIRT7 is involved in HG-induced podocyte apoptosis. To investigate whether SIRT7 is involved in HG-induced podocyte apoptosis, the present study investigated the effect of SIRT7 knockdown or SIRT7 overexpression on podocyte apoptosis. For knockdown of SIRT7, podocytes were transfected with SIRT7 siRNA. The results demonstrated that transfection with SIRT7 siRNA significantly decreased the protein expression of SIRT7 in HG-treated cells (Fig. 5A). Furthermore, knockdown of SIRT7 significantly increased podocyte apoptosis induced by HG (Fig. 5B). Conversely, SIRT7 overexpression led to significantly increased protein levels of SIRT7 in HG-treated cells (Fig. 5C) and markedly inhibited podocyte 
A

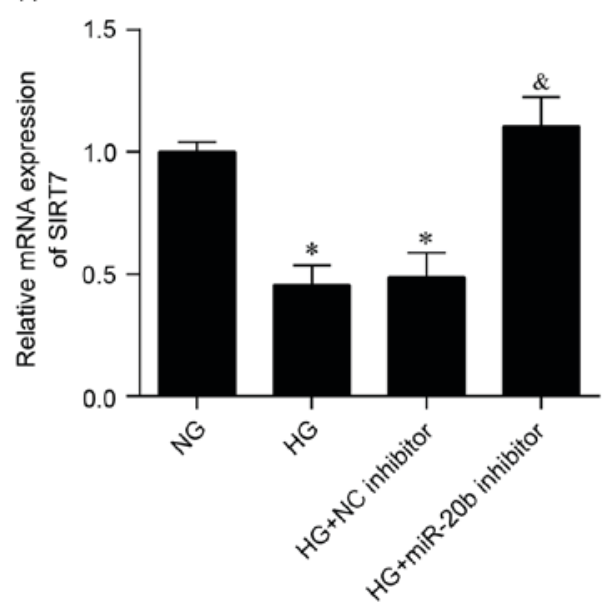

B

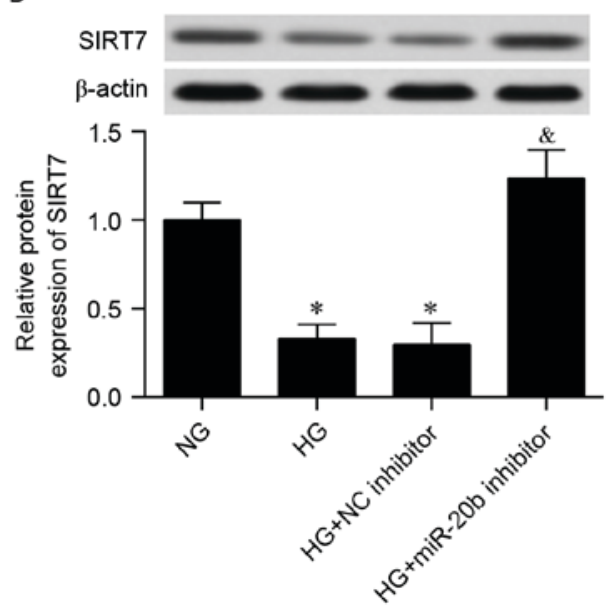

Figure 4. Suppression of miR-20b upregulates SIRT7 expression. The (A) mRNA and (B) protein expression of SIRT7 in NG (5 mM) and HG (30 mM)-treated cells with or without transfection with miR-20b inhibitor or NC inhibitor was detected by reverse transcription-quantitative polymerase chain reaction and western blot analysis, respectively. ${ }^{*} \mathrm{P}<0.05$ vs. NG; ${ }^{\circledR} \mathrm{P}<0.05$ vs. HG and HG + NC inhibitor. miR, microRNA; SIRT7, sirtuin 7; NG, normal glucose; HG, high glucose; NC, negative control.

A
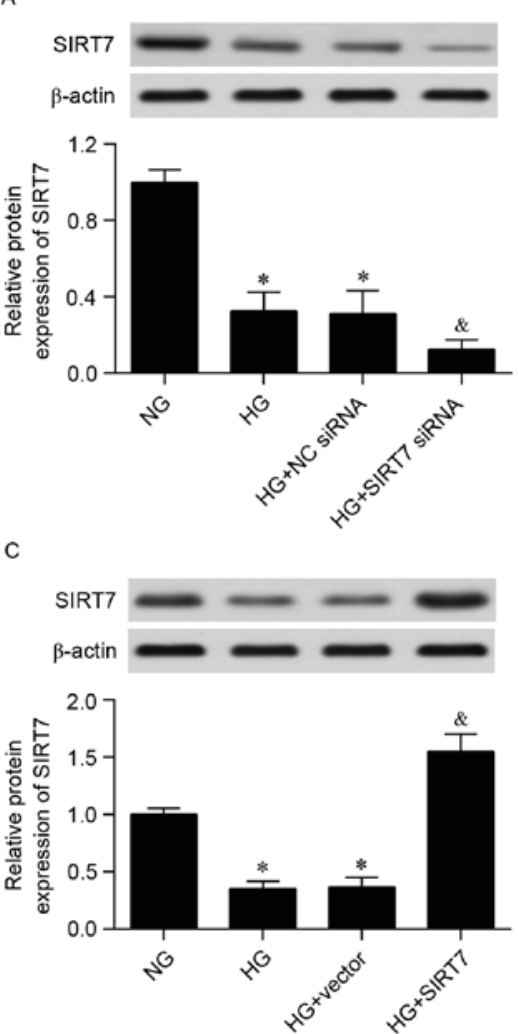
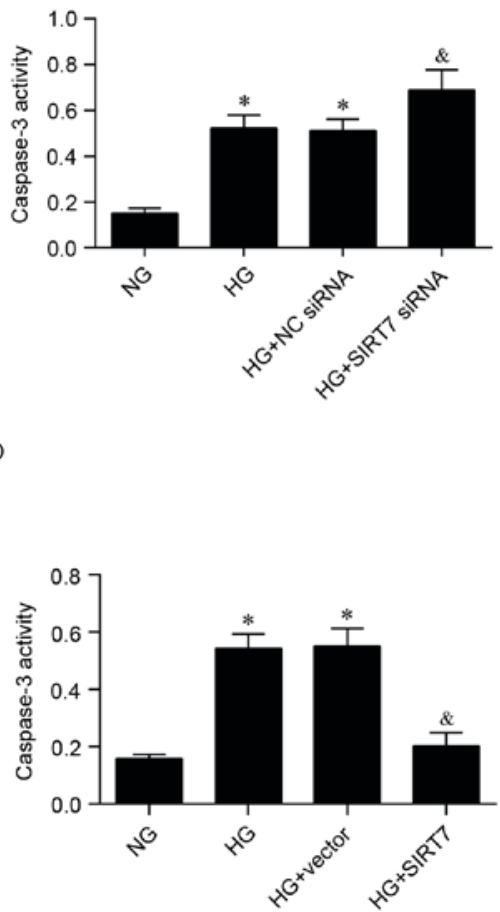

Figure 5. SIRT7 is involved in HG-induced podocyte apoptosis. Podocytes were treated with NG $(5 \mathrm{mM})$ or HG $(30 \mathrm{mM})$ with or without transfection with SIRT7 siRNA or NC siRNA. (A) Western blot analysis of SIRT7 protein expression in podocytes transfected with SIRT7 siRNA or NC siRNA for $24 \mathrm{~h}$. (B) Effect of SIRT7 knockdown on podocyte apoptosis was detected by a caspase-3 activity assay. " P $<0.05$ vs. NG; ${ }^{\circledR}$ P $<0.05$ vs. HG and HG + NC siRNA. Podocytes were treated with NG $(5 \mathrm{mM})$ or $\mathrm{HG}(30 \mathrm{mM})$ with or without transfection with SIRT7 overexpression vector or control vector. (C) Western blot analysis of SIRT7 protein expression in podocytes transfected with pcDNA3.1/SIRT7 overexpression vector or empty vector for $24 \mathrm{~h}$. (D) Effect of SIRT7 overexpression on podocyte apoptosis was detected by a caspase-3 activity assay. ${ }^{*} \mathrm{P}<0.05$ vs. NG; ${ }^{2} \mathrm{P}<0.05$ vs. HG and HG + vector. SIRT7, sirtuin 7; HG, high glucose; NG, normal glucose; siRNA, small interfering RNA; NC, negative control; HG + vector, HG-treated cells transfected with empty vector; HG + SIRT7, HG-treated cells transfected with pcDNA3.1/SIRT7 overexpression vector.

apoptosis induced by HG (Fig. 5D), which mimicked the effect of miR-20b suppression. These results indicate that SIRT7 may be involved in the protection of podocytes from HG-induced apoptosis.
Knockdown of SIRT7 blocks the protective effect of $m i R-20 \mathrm{~b}$ suppression. To verify whether SIRT7 is involved in the observed miR-20b-mediated protective effect, podocytes were cotransfected with miR-20b inhibitor and SIRT7 


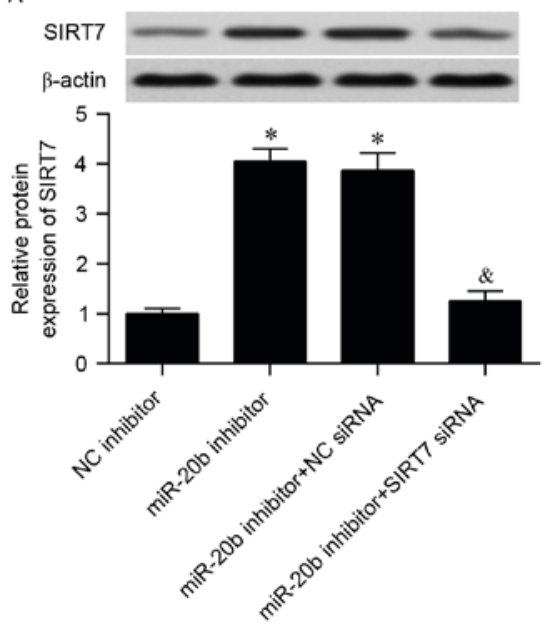

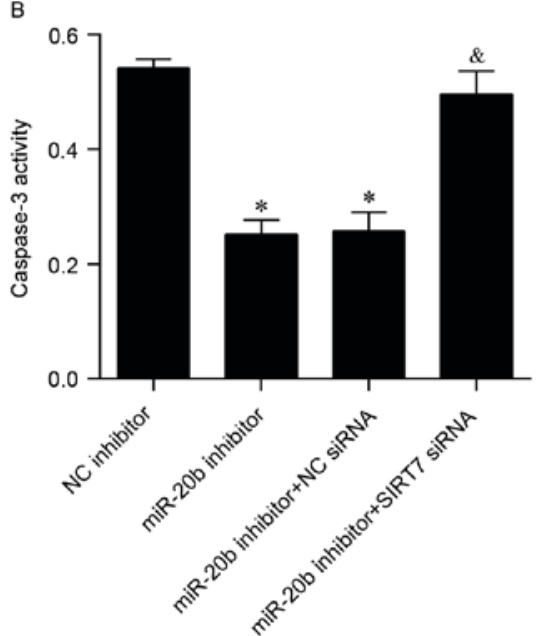

Figure 6. Knockdown of SIRT7 blocks the protective effect of miR-20b suppression. Cells were treated with high glucose (30 mM) and transfected with NC inhibitor or miR-20b inhibitor, or cotransfected with miR-20b inhibitor and NC or SIRT7 siRNA. (A) Western blot analysis was performed to investigate the protein expression of SIRT7 in podocytes cotransfected with miR-20b inhibitor and SIRT7 siRNA. (B) Podocyte apoptosis was measured using a caspase-3 activity assay. "P $<0.05$ vs. NC inhibitor; ${ }^{\circledR} \mathrm{P}<0.05$ vs. miR-20b inhibitor and miR-20b inhibitor + NC siRNA. SIRT7, sirtuin 7; miR, microRNA; NC, negative control; siRNA, small interfering RNA.

siRNA. The results demonstrated that the increase in SIRT7 expression following miR-20b suppression was significantly reduced by SIRT7 knockdown (Fig. 6A). In addition, the protective effect of miR-20b suppression against HG-induced apoptosis was significantly blocked by SIRT7 knockdown (Fig. 6B).

\section{Discussion}

Previous evidence has indicated that miRNAs have an important role in DN and that miRNAs may serve as diagnosis biomarkers and therapeutic candidates $(14,15)$. Therefore, the identification of DN-associated miRNAs is essential for the development of novel therapeutic strategies. The present study, to the best of our knowledge, is the first to demonstrate that miR-20b may be a hyperglycemia-responsive miRNA as it was induced by $\mathrm{HG}$ in podocytes and suppression of miR-20b provided a protective effect against HG-induced apoptosis. The present study also identified that SIRT7 is a functional target of miR-20b that contributed to the miR-20b suppression-mediated protective effect against apoptosis. In conclusion, the results of the present study revealed a novel miRNA-based mechanism for regulating podocyte apoptosis.

An increasing number of studies have reported important roles for miRNAs in the regulation of podocyte apoptosis (28). Among these, certain studies have concluded that HG-induced podocyte apoptosis was attenuated by miR-29a overexpression (29) and overexpression of miR-29c strongly induced podocyte apoptosis by targeting sprouty homolog 1 (30). In addition, Chen et al (31) reported that miR-195 was increased in diabetic mice and promoted podocyte apoptosis induced by HG by inhibiting Bcl-2. Recently, miR-34c and miR-218 were reported to contribute to HG-induced podocyte apoptosis by targeting Notch signaling or heme oxygenase-1, respectively $(16,32)$. The present study demonstrated that miR-20b was significantly induced by HG in cultured podocytes and suppression of miR-20b provided a protective effect against
HG. The current study has identified a potential novel regulator of podocyte apoptosis.

Previous reports have demonstrated that miR-20b is extensively involved in several pathological processes $(33,34)$. The inhibition of oncogenes in various cancer cell lines has revealed an antiproliferative properties of miR-20b (35-37). In addition, suppression of miR-20b provided cardioprotection against ischemia/reperfusion injury (38), and overexpression of miR-20b promoted apoptosis of P19 cells by inducing mitochondrial impairment (24). The present study demonstrated that miR-20b contributed to HG-induced podocyte apoptosis, further confirming the proapoptotic role of miR-20b. However, the underlying mechanism of miR-20b in regulating apoptosis remains to be elucidated.

The current study identified that SIRT7 is a functional target of miR-20b in the regulation of podocyte apoptosis. SIRT7 is the seventh member of the sirtuin family and has an important role in cell proliferation, stress and disease (20). Cumulative evidence has indicated that SIRT7 functions as an important regulator of cell survival and apoptosis $(20,23,39)$. SIRT7 was also reported to prevent cardiomyocyte apoptosis by inhibiting the p53 proapoptotic signaling pathway (40), and promote myocardial tissue repair following myocardial infarction and hind-limb ischemia (41). Furthermore, knockdown of SIRT7 induced gastric cancer cell apoptosis (39), and promoted cellular survival and inhibited apoptosis induced by genomic stress via repression of stress-activated kinases (p38 and c-Jun N-terminal kinase) and the p53 signaling pathway $(23,42)$. SIRT7 was also reported to have a vital role in sensing cellular energy levels and conserving energy during stress (43). Thus, SIRT7 aids cellular survival in response to stress conditions. The present study demonstrated that SIRT7 is also an important regulator under HG conditions. The results indicated that SIRT7 overexpression protected podocytes against HG-induced apoptosis, while SIRT7 silencing promoted apoptosis, indicating that SIRT7 may promote the survival of podocytes under HG conditions. 
Regulation of SIRT7 by specific miRNAs has been reported by recent studies $(44,45)$. Several studies have revealed that miR-125b inhibits cancer development by targeting SIRT7 in bladder cancer $(46)$ and hepatocellular carcinoma $(47,48)$. In addition, suppression of SIRT7 by miR-3666 induced cell apoptosis of non-small cell lung cancer (49), and Gu et al (50) reported that miR-152 induced human dental pulp stem cell senescence by targeting SIRT7. The results of the current study indicated that miR-20b may be a novel regulator of SIRT7, indicating that SIRT7 undergoes epigenetic regulation by various miRNAs, which may exert effects in various pathological processes.

To the best of our knowledge, the present study is the first to demonstrate that miR-20b may be an important regulator of HG-induced podocyte apoptosis. The results demonstrated that SIRT7 is a direct target of miR-20b in the regulation of podocyte apoptosis. The results of the current study may provide novel insight into the effects of miR-20b in DN. Therefore, miR-20b may serve as a promising therapeutic target for the treatment of DN. However, further in vitro and in vivo studies are required to fully elucidate the precise role and molecular mechanism of miR-20b in DN.

\section{References}

1. Nakai S, Masakane I, Akiba T, Shigematsu T, Yamagata K, Watanabe Y, Iseki K, Itami N, Shinoda T, Morozumi K, et al: Overview of regular dialysis treatment in Japan as of 31 December 2006. Ther Apher Dial 12: 428-456, 2008.

2. Yoon KH, Lee JH, Kim JW, Cho JH, Choi YH, Ko SH, Zimmet P and Son HY: Epidemic obesity and type 2 diabetes in Asia. Lancet 368: 1681-1688, 2006.

3. Shankland SJ: The podocyte's response to injury: Role in proteinuria and glomerulosclerosis. Kidney Int 69: 2131-2147, 2006.

4. Coimbra TM, Janssen U, Gröne HJ, Ostendorf T, Kunter U, Schmidt H, Brabant G and Floege J: Early events leading to renal injury in obese Zucker (fatty) rats with type II diabetes. Kidney Int 57: 167-182, 2000.

5. Siu B, Saha J, Smoyer WE, Sullivan KA and Brosius FC III: Reduction in podocyte density as a pathologic feature in early diabetic nephropathy in rodents: Prevention by lipoic acid treatment. BMC Nephrol 7: 6, 2006.

6. Steffes MW, Schmidt D, McCrery R and Basgen JM; International Diabetic Nephropathy Study Group: Glomerular cell number in normal subjects and in type 1 diabetic patients. Kidney Int 59: 2104-2113, 2001

7. Susztak K, Raff AC, Schiffer M and Böttinger EP: Glucose-induced reactive oxygen species cause apoptosis of podocytes and podocyte depletion at the onset of diabetic nephropathy. Diabetes 55: 225-233, 2006.

8. Bartel DP: microRNAs: Genomics, biogenesis, mechanism, and function. Cell 116: 281-297, 2004.

9. Winter J, Jung S, Keller S, Gregory RI and Diederichs S: Many roads to maturity: microRNA biogenesis pathways and their regulation. Nat Cell Biol 11: 228-234, 2009.

10. Mendell JT and Olson EN: microRNAs in stress signaling and human disease. Cell 148: 1172-1187, 2012.

11. Piletič $K$ and Kunej T: microRNA epigenetic signatures in human disease. Arch Toxicol 90: 2405-2419, 2016.

12. Jones Buie JN, Goodwin AJ, Cook JA, Halushka PV and Fan H: The role of miRNAs in cardiovascular disease risk factors. Atherosclerosis 254: 271-281, 2016.

13. Volný $\mathrm{O}$, Kašičková L, Coufalová $\mathrm{D}$, Cimflová $\mathrm{P}$ and Novák J: microRNAs in cerebrovascular disease. Adv Exp Med Biol 888: 155-195, 2015.

14. Simpson K, Wonnacott A, Fraser DJ and Bowen T: microRNAs in diabetic nephropathy: From biomarkers to therapy. Curr Diab Rep 16: 35, 2016.

15. Kato $M$ and Natarajan R: microRNAs in diabetic nephropathy: Functions, biomarkers, and therapeutic targets. Ann N Y Acad Sci 1353: 72-88, 2015.
16. Liu XD, Zhang LY, Zhu TC, Zhang RF, Wang SL and Bao Y: Overexpression of miR-34c inhibits high glucose-induced apoptosis in podocytes by targeting Notch signaling pathways. Int $\mathrm{J}$ Clin Exp Pathol 8: 4525-4534, 2015.

17. Xie H, Lin HL, Wang N, Sun YL, Kan Y, Guo H, Chen JL and Fang M: Inhibition of microRNA-30a prevents puromycin aminonucleoside-induced podocytic apoptosis by upregulating the glucocorticoid receptor $\alpha$. Mol Med Rep 12: 6043-6052, 2015 .

18. Houtkooper RH, Pirinen E and Auwerx J: Sirtuins as regulators of metabolism and healthspan. Nat Rev Mol Cell Biol 13: 225-238, 2012.

19. Michishita E, Park JY, Burneskis JM, Barrett JC and Horikawa I: Evolutionarily conserved and nonconserved cellular localizations and functions of human SIRT proteins. Mol Biol Cell 16: 4623-4635, 2005.

20. Kiran S, Anwar T, Kiran M and Ramakrishna G: Sirtuin 7 in cell proliferation, stress and disease: Rise of the seventh sirtuin! Cell Signal 27: 673-682, 2015.

21. Hubbi ME, Hu H, Kshitiz, Gilkes DM and Semenza GL: Sirtuin-7 inhibits the activity of hypoxia-inducible factors. J Biol Chem 288: 20768-20775, 2013.

22. Shin J, He M, Liu Y, Paredes S, Villanova L, Brown K, Qiu X, Nabavi N, Mohrin M, Wojnoonski K, et al: SIRT7 represses Myc activity to suppress ER stress and prevent fatty liver disease. Cell Rep 5: 654-665, 2013.

23. Kiran S, Oddi V and Ramakrishna G: Sirtuin 7 promotes cellular survival following genomic stress by attenuation of DNA damage, SAPK activation and p53 response. Exp Cell Res 331: 123-141, 2015.

24. Zhu S, Hu X, Yu Z, Peng Y, Zhu J, Liu X, Li M, Han S and Zhu C: Effect of miR-20b on apoptosis, differentiation, the BMP signaling pathway and mitochondrial function in the P19 cell model of cardiac differentiation in vitro. PLoS One 10: e0123519, 2015.

25. Qin B, Liu J, Liu S, Li B and Ren J: miR-20b targets AKT3 and modulates vascular endothelial growth factor-mediated changes in diabetic retinopathy. Acta Biochim Biophys Sin (Shanghai) 48: 732-740, 2016.

26. Mundel P, Reiser J, Zúñiga Mejía Borja A, Pavenstädt $H$, Davidson GR, Kriz W and Zeller R: Rearrangements of the cytoskeleton and cell contacts induce process formation during differentiation of conditionally immortalized mouse podocyte cell lines. Exp Cell Res 236: 248-258, 1997.

27. Livak KJ and Schmittgen TD: Analysis of relative gene expression data using real-time quantitative PCR and the 2(-Delta Delta C(T)) method. Methods 25: 402-408, 2001.

28. Li JY, Yong TY, Michael MZ and Gleadle JM: Review: The role of microRNAs in kidney disease. Nephrology (Carlton) 15: 599-608, 2010.

29. Lin CL, Lee PH, Hsu YC, Lei CC, Ko JY, Chuang PC, Huang YT, Wang SY, Wu SL, Chen YS, et al: microRNA-29a promotion of nephrin acetylation ameliorates hyperglycemia-induced podocyte dysfunction. J Am Soc Nephrol 25: 1698-1709, 2014.

30. Long J, Wang Y, Wang W, Chang BH and Danesh FR: microRNA-29c is a signature microRNA under high glucose conditions that targets Sprouty homolog 1, and its in vivo knockdown prevents progression of diabetic nephropathy. J Biol Chem 286: 11837-11848, 2011.

31. Chen YQ, Wang XX, Yao XM, Zhang DL, Yang XF, Tian SF and Wang NS: microRNA-195 promotes apoptosis in mouse podocytes via enhanced caspase activity driven by BCL2 insufficiency. Am J Nephrol 34: 549-559, 2011.

32. Yang H, Wang Q and Li S: microRNA-218 promotes high glucose-induced apoptosis in podocytes by targeting heme oxygenase-1. Biochem Biophys Res Commun 471: 582-588, 2016.

33. Chunjie N, Huijuan N, Zhao Y, Jianzhao W and Xiaojian Z: Disease-specific signature of serum miR-20b and its targets IL-8 and IL-25, in myasthenia gravis patients. Eur Cytokine Netw 26: 61-66, 2015.

34. Ingwersen J, Menge $\mathrm{T}$, Wingerath $\mathrm{B}$, Kaya D, Graf J, Prozorovski T, Keller A, Backes C, Beier M, Scheffler M, et al: Natalizumab restores aberrant miRNA expression profile in multiple sclerosis and reveals a critical role for miR-20b. Ann Clin Transl Neurol 2: 43-55, 2015.

35. Khuu C, Sehic A, Eide L and Osmundsen H: Anti-proliferative properties of miR-20b and miR-363 from the miR-106a-363 cluster on human carcinoma cells. Microrna 5: 19-35, 2016. 
36. Liu M, Wang D and Li N: microRNA-20b downregulates HIF-10 and inhibits the proliferation and invasion of osteosarcoma cells. Oncol Res 23: 257-266, 2016.

37. Park SL, Cho TM, Won SY, Song JH, Noh DH, Kim WJ and Moon SK: microRNA-20b inhibits the proliferation, migration and invasion of bladder cancer EJ cells via the targeting of cell cycle regulation and Sp-1-mediated MMP-2 expression. Oncol Rep 34: 1605-1612, 2015.

38. Mukhopadhyay P, Das S, Ahsan MK, Otani H and Das DK: Modulation of microRNA 20b with resveratrol and longevinex is linked with their potent anti-angiogenic action in the ischaemic myocardium and synergestic effects of resveratrol and $\gamma$-tocotrienol. J Cell Mol Med 16: 2504-2517, 2012.

39. Zhang S, Chen P, Huang Z, Hu X, Chen M, Hu S, Hu Y and Cai T: Sirt7 promotes gastric cancer growth and inhibits apoptosis by epigenetically inhibiting miR-34a. Sci Rep 5: 9787, 2015.

40. Vakhrusheva O, Smolka C, Gajawada P, Kostin S, Boettger T, Kubin T, Braun T and Bober E: Sirt7 increases stress resistance of cardiomyocytes and prevents apoptosis and inflammatory cardiomyopathy in mice. Circ Res 102: 703-710, 2008.

41. Araki S, Izumiya Y, Rokutanda T, Ianni A, Hanatani S, Kimura Y, Onoue Y, Senokuchi T, Yoshizawa T, Yasuda O, et al: Sirt7 contributes to myocardial tissue repair by maintaining transforming growth factor- $\beta$ signaling pathway. Circulation 132: 1081-1093, 2015.

42. Nahálková J: Novel protein-protein interactions of TPPII, p53, and SIRT7. Mol Cell Biochem 409: 13-22, 2015.

43. Chen S, Seiler J, Santiago-Reichelt M, Felbel K, Grummt I and Voit R: Repression of RNA polymerase I upon stress is caused by inhibition of RNA-dependent deacetylation of PAF53 by SIRT7. Mol Cell 52: 303-313, 2013.
44. CioffiM,Vallespinos-SerranoM,TrabuloSM,Fernandez-MarcosPJ, Firment AN, Vazquez BN, Vieira CR, Mulero F, Camara JA, Cronin UP, et al: miR-93 controls adiposity via inhibition of Sirt7 and Tbx3. Cell Rep 12: 1594-1605, 2015.

45. Kurylowicz A, Owczarz M, Polosak J, Jonas MI, Lisik W, Jonas M, Chmura A and Puzianowska-Kuznicka M: SIRT1 and SIRT7 expression in adipose tissues of obese and normal-weight individuals is regulated by microRNAs but not by methylation status. Int J Obes (Lond) 40: 1635-1642, 2016.

46. Han Y, Liu Y, Zhang H, Wang T, Diao R, Jiang Z, Gui Y and Cai Z: Hsa-miR-125b suppresses bladder cancer development by down-regulating oncogene SIRT7 and oncogenic long non-coding RNA MALAT1. FEBS Lett 587: 3875-3882, 2013.

47. Kim JK, Noh JH, Jung KH, Eun JW, Bae HJ, Kim MG, Chang YG, Shen Q, Park WS, Lee JY, et al: Sirtuin7 oncogenic potential in human hepatocellular carcinoma and its regulation by the tumor suppressors miR-125a-5p and miR-125b. Hepatology 57: 1055-1067, 2013

48. Zhao L and Wang W: miR-125b suppresses the proliferation of hepatocellular carcinoma cells by targeting Sirtuin7. Int J Clin Exp Med 8: 18469-18475, 2015.

49. Shi H, Ji Y, Zhang D, Liu Y and Fang P: microRNA-3666-induced suppression of SIRT7 inhibits the growth of non-small cell lung cancer cells. Oncol Rep 36: 3051-3057, 2016.

50. Gu S, Ran S, Liu B and Liang J: miR-152 induces human dental pulp stem cell senescence by inhibiting SIRT7 expression. FEBS Lett 590: 1123-1131, 2016. 